\title{
El trabajo con proyectos en las universidades pedagógicas cubanas: herramienta para el logro del modo de actuación hacia la actividad investigativa
}

\section{Project work at Cuban Pedagogical Universities: tool for managing the research performance}

\author{
Deysi Fernández* \\ Sirelda García** \\ Diana Morales***
}

\section{Resumen}

La política educacional cubana ha estado sustentada en una concepción pedagógica dirigida conscientemente hacia la formación científica y tecnológica de la juventud para garantizar el desarrollo de la nación, frente a un mundo hostil y competitivo. Es por ello que el futuro profesional de la educación se prepara desde su formación inicial para enfrentar los retos del desarrollo actual y futuro.

La realización de proyectos por el profesor en formación de lenguas extranjeras, particularmente desde las disciplinas Práctica Integral de la Lengua Inglesa y Práctica Integral de la Lengua Francesa, constituye una herramienta importante para contribuir a la preparación de las nuevas generaciones de profesores en el desarrollo de la competencia comunicativa y particularmente en la formación de modos de actuación hacia la actividad investigativa como futuros profesionales. Estos proyectos les permiten desarrollar las habilidades atinentes al dominio de los métodos de la investigación científica y cualidades tales como creatividad, autoformación y autovaloración.

La experiencia con la realización de proyectos con los estudiantes de los tres primeros años de la carrera ha permitido desarrollar de forma paralela la competencia comunicativa y habilidades investigativas al indagar, hablar y escribir sobre diferentes temáticas entre las que se destacan: Educación, Deporte, Medicina, Medioambiente, Discriminación de la mujer, Salud (Tabaquismo y Alcoholismo), Sexualidad y Costumbres y Tradiciones. La experiencia ha permitido constatar fortalezas y debilidades que guían el trabajo para el logro del objetivo trazado.

PALABRAS CLAVE: proyectos, educación, profesor en formación, lenguas extranjeras, investigación, modo de actuación

\begin{abstract}
The Cuban educational policy is based on a pedagogical conception consciously oriented to the scientific and technological formation of the young generations in order to guarantee the development of the nation in a hostile and competitive world. That is why pre-service teachers majoring in Pedagogy are well prepared from their initial professional formation to face the present and future challenges in this sphere. Project work in Integrated English Practice and in Integrated French Practice has been an important tool to contribute to the development of the communicative competence and to the scientific formation of pre-service teachers majoring in Foreign Language Teaching. They particularly contribute to the formation of modes
\end{abstract}


of performance regarding research. Such projects allow the development of abilities for the mastering of scientific research methods and qualities such as creativity, self-formation and self-assessment.

The experience regarding the effectiveness of project work carried out with the 1 st, 2nd and $3 r d$ year students has permitted the development of the communicative competence while investigating about different subjects such as Education, Sports, Medicine, Environment, Women discrimination, Health (tabaquism and alcoholism), Sexuality, Customs and traditions. The experience has permitted to identify weaknesses and strengths that guide the work to achieve the goal proposed.

KEY WORDS: project work, education, pre-service teacher, foreign languages, research, mode of performance

\section{Introducción}

"Al venir a la tierra, todo hombre tiene derecho a que se le eduque, y después, en pago, el deber de contribuir a la educación de los demás". 1

El aprendizaje de al menos un idioma extranjero como parte de la educación general de la población cubana es una necesidad social para el desarrollo económico y cultural de nuestro país, así como para el establecimiento de relaciones con otras naciones.

La enseñanza del inglés como lengua extranjera se ha privilegiado ya que se comienza a impartir desde el tercer grado en la enseñanza primaria y transita por todos los grados y niveles. Ésta ha estado signada en la última década por el afianzamiento de un enfoque comunicativo donde la motivación de los estudiantes es de primordial importancia en la adquisición de conocimientos y habilidades. El aprendizaje ocurre cuando el contenido se relaciona con las necesidades y experiencias de los estudiantes y por tanto es significativo en su vida o en situaciones similares, y la participación de los estudiantes es activa en el proceso de aprendizaje requiriendo en ocasiones de niveles de ayuda para percibir las relaciones entre los elementos en el idioma y en diferentes situaciones culturales.

La carrera en Licenciatura en Educación especialidad Lenguas Extranjeras (Inglés con Segunda Lengua) da respuesta al reclamo de que la población cubana domine un mayor número de lenguas como instrumentos indispensables para el acceso a la información y la comunicación, con el objetivo de incrementar el intercambio entre los pueblos ante la creciente necesidad de aunar esfuerzos para resolver los acuciantes problemas de la humanidad. Esta carrera cobra mayor pertinencia social en los momentos actuales debido a la necesidad de facilitar las relaciones de cooperación con otros pueblos, propósito para el que la formación de profesores de lenguas extranjeras es esencial. La carrera ha transitado por procesos de perfeccionamiento en correspondencia con las exigencias del desarrollo socio-histórico y con las demandas del contexto mundial y se ha caracterizado hasta el presente por profundas transformaciones.

La Universidad de Ciencias Pedagógicas "Félix Varela Morales" es una de las universidades del país que se acoge a la variante de formación de un profesor de lenguas extranjeras (inglés con segunda lengua) reconociendo como uno de sus retos insoslayables la elevación de la gestión de la calidad de los procesos de formación que en ella se dan. En la búsqueda de esta mejora se trabaja en las vías que permitan al profesor en formación desarrollar modos de actuación para responder en su desempeño profesional al perfil amplio para el que se forma. 
Aunque se logran avances en la formación de estos profesionales aún se identifican como debilidades el bajo nivel de desarrollo de la competencia comunicativa en las lenguas extranjeras en algunas cohortes y el limitado logro de modos de actuación profesional hacia la investigación que se correspondan con las necesidades que el momento y el futuro del país demandan.

El presente trabajo investigativo resalta las ventajas que ofrece el trabajo con proyectos en la enseñanza de las lenguas extranjeras, particularmente la experiencia de la enseñanza del inglés y del francés para contribuir al desarrollo de la competencia comunicativa y a modo de actuación profesional hacia la investigación educativa en la preparación del profesor en formación de lenguas extranjeras.

\section{Desarrollo}

La enseñanza de las lenguas extranjeras en las universidades pedagógicas cubanas sigue el enfoque comunicativo que tiene como objetivo supremo el desarrollo de la competencia comunicativa en sus futuros profesionales. Este enfoque aboga por más oportunidades para integrar todo lo aprendido y estimular la comunicación genuina. Con él se ha privilegiado el trabajo con actividades tanto orales como escritas, actividades de lectura, de audición, el trabajo en equipos, y otras que estimulan en los estudiantes la reflexión, el desarrollo de estrategias para el aprendizaje, el pensamiento lógico, la creatividad, el pensamiento crítico y la cooperación. Sin embargo, un profesional que se forme para el perfil educacional debe prepararse no solo para lograr los niveles de competencia comunicativa requeridos en el idioma que estudia sino también debe desarrollar modos de actuación que le permitan desempeñarse exitosamente en su futura profesión. Como ha expresado un destacado pedagogo cubano, además de instruir al estudiante durante su formación resulta igualmente necesario ponerlo en contacto con el objeto de su profesión desde los primeros años de la carrera, y así lograr el imprescindible nexo con los modos de actuación de esa profesión desde sus aspectos más simples y elementales, hasta aquellos más complejos y que demandan mayor nivel de preparación. Solo de ese modo se aseguran las habilidades necesarias para su desempeño profesional (Horruitiner Silva: 19).

El desarrollo de modos de actuación para investigar en su propia práctica es esencial en un profesional competente. Sin embargo, se ha identificado como uno de los problemas más complejos que en la actualidad enfrentan las instituciones que forman profesores el logro en los futuros profesionales de la educación de una cultura científico-investigativa que les permita desarrollar una actividad profesional reflexiva, crítica y transformadora de su práctica (Salazar D.,) 2007: 177).

A pesar de la introducción de cambios novedosos en la enseñanza de las lenguas extranjeras, los resultados que se obtienen tanto en el desarrollo de la competencia comunicativa como en los modos de actuación hacia la investigación aún no alcanzan los niveles a los que se aspira. La situación anteriormente expuesta ha motivado a los profesores de las disciplinas Práctica Integral de la Lengua Inglesa y Práctica Integral de la Lengua Francesa de la Universidad de Ciencias Pedagógicas "Félix Varela Morales" a poner en práctica diferentes actividades que permitan la formación de un profesional más competente, capaz de resolver los problemas profesionales de manera creativa con un adecuado equilibrio entre la competencia comunicativa y los modos de actuación investigativos. Una de las actividades para el logro de dicho objetivo, de gran aceptación entre profesores y estudiantes debido a su efectividad, es el trabajo con proyectos. 
El trabajo con los proyectos acerca a los estudiantes al proceso investigativo y de esta forma, como plantearan clásicos de la pedagogía cubana entre los que se destacan José Martí, José de la Luz y Caballero y Enrique José Varona el maestro no solo convierte su clase en un acto cercano a la investigación científica sino que puede y debe investigar desde su aula (Chávez, J. Video clases de MIE 1ro).

También profesionales cubanos como Núñez (1999) y Lage (1996) (citados en Salazar D, 2007:179) han coincidido al plantear que el pensamiento científico debe pasar a formar parte de la cultura profesional e ir aproximando las fronteras entre la formación científico-investigativa y la formación cultural general a la que se aspira en los docentes en formación.

La atención al desarrollo de las habilidades investigativas en las disciplinas Práctica Integral de la Lengua Inglesa y Práctica Integral de la Lengua Francesa ha estado muy vinculada a la filosofía seguida en la enseñanza de una lengua extranjera en Cuba.

A partir de la década del 90, la educación cubana incursiona en la enseñanza comunicativa del idioma. Sobre esta temática se han pronunciado profesores cubanos como Enríquez O'Farril I. (1999), Camacho Delgado A. (2004) y Font Sergio (2006), quienes coinciden en afirmar que ha habido desde entonces un proceso de cambio sustantivo en el que se asume una concepción comunicativa y desarrolladora dejando atrás la visión estructuralista y tradicional que la lastraba. La nueva concepción en la enseñanza de idiomas permite la aplicación de diferentes principios para la enseñanza comunicativa definidos por varios estudiosos, entre los que se destacan Neil Naiman, Keith Morrow, Alice Omaggio y Alfredo Camacho Delgado.La materialización de estos postulados favorece además el desarrollo de habilidades investigativas. Tal es el caso del principio que plantea - la clase centrada en el estudiante- por cuanto el alumno se involucra activamente en el proceso de aprendizaje, acción necesaria para que el profesor en formación se convierta en un profesional investigador de su propia práctica ; el desarrollo de estrategias para continuar desarrollando habilidades fuera del aula, fundamentalmente aquellas que favorecen a la lectura y escritura; el todo es más importante que la suma de sus partes, que ayuda al estudiante a identificar y trabajar con las ideas fundamentales del texto para luego resumir o parafrasear según sea la necesidad; el establecimiento de relaciones interdisciplinarias, principio que ofrece al alumno oportunidades para aprender contenidos de otras materias del currículo escolar a través de la lengua extranjera.

\section{Los proyectos como vía esencial para el objetivo trazado}

Varios han sido los especialistas que han reconocido el valor de este tipo de actividad particularmente en lo referido a su aporte al desarrollo de habilidades investigativas.

El proyecto constituye un medio fundamental por el cual se forman y desarrollan habilidades investigativas tales como: identificar un problema, planificar un proceso investigativo, ejecutar lo planificado, evaluar el impacto de la solución del problema de acuerdo con lo planificado y comunicar los resultados en un proceso de construcción constante de aprendizajes (Medina, J 2011:33).

Por su parte, el Centro de Estudios Para el Aprendizaje (CEPAP)- UNESR define el proyecto de aprendizaje como un conjunto de acciones planificadas y desarrolladas por una persona o grupo, tanto para obtener beneficios socio-educativos como para lograr un conjunto de aprendizajes definidos. 
La inclusión de los proyectos en la enseñanza del inglés con un enfoque comunicativo a partir de la segunda mitad de la década de los 80 se encaminó fundamentalmente hacia la formación de una cultura general integral en los estudiantes, hacia una integración de habilidades de manera natural y como un preámbulo para la escritura de los trabajos de diploma y de curso (Morales, A. y otros, 1998: 33). La realización de proyectos de aprendizaje es considerada una vía esencial para el desarrollo de habilidades comunicativas e investigativas ya que dichos proyectos consisten en actividades comunicativo-conclusivas centradas en la realización de tareas individuales y/o grupales tanto dentro como fuera del aula que promueven el aprendizaje cooperativo y permiten, tal como se dijo anteriormente, el desarrollo de habilidades comunicativas sociales, de planificación, control y evaluación en correspondencia con el nivel de competencia comunicativa que se requiere para cada nivel (Morales D, 2005: 13).

Las definiciones anteriores coinciden en señalar a los proyectos como tareas que requieren de trabajo independiente por parte del estudiante y que promueven el aprendizaje cooperativo que permite desarrollar habilidades comunicativas al realizar entrevistas y reportar sobre el trabajo realizado; habilidades investigativas, al leer o indagar sobre el tema que desarrollan y al procesar la información recopilada; y habilidades sociales, pues durante el proyecto los estudiantes realizan debates, colaboran con otros compañeros y conocen a nuevas personas.

Destacados profesionales de la enseñanza de las lenguas extranjeras se han referido a las principales características de los proyectos. Entre ellos sobresalen Carter y Thomas (1986), Ferragatti y Carminati (1984), Fried-Booth (1982, 1986), Haines (1989), Legutke (1984, 1985), Legutke and Thied (1983), Papandreou (1994), Sheppard and Stroller (1995) y Ward (1998) quienes, a pesar de aproximarse al trabajo con los proyectos desde diferentes perspectivas, han coincidido en las siguientes características esenciales:

1. El trabajo con los proyectos se centra en el aprendizaje de contenidos más que en aspectos específicos del idioma, lo que significa que los proyectos se pueden centrar en temas actuales y tópicos de interés para los estudiantes.

2. El trabajo con los proyectos tiene esencialmente como centro a los estudiantes, aunque el profesor juega un rol importante al ofrecer apoyo y ser guía a través de todo el proceso de desarrollo del proyecto.

3. El trabajo con los proyectos es cooperativo y no competitivo por lo que los estudiantes pueden trabajar tanto de forma individual como en pequeños grupos o toda el aula para realizar un proyecto, compartir recursos, ideas y experiencias a lo largo del mismo.

4. El trabajo con los proyectos permite una auténtica integración de habilidades y procesamiento de informaciones de diferentes fuentes.

5. El trabajo con los proyectos culmina con un producto final, e.g.: presentación oral, despliegue de carteles, murales, reportes o actuaciones, que puedan ser compartidos con otros y por ende los proyectos tienen propósitos reales, aunque el valor de los proyectos radica más en el proceso que se despliega para lograr el objetivo que en el producto en sí. Por ende, los proyectos tienen una orientación de proceso y producto a la vez y brindan a los estudiantes oportunidades para centrarse tanto en la fluidez (fluency) como en el uso adecuado del idioma o precisión (accuracy) a lo largo de su desarrollo. 
6. El trabajo con los proyectos es en sí motivador, desarrollador y cambiante. El resultado que provoca en los estudiantes es positivo pues éstos aumentan su autoestima, confianza y autonomía a la vez que mejoran sus habilidades en el idioma, el aprendizaje de contenidos de otras áreas del currículo escolar y de habilidades cognitivas.

Dentro de los propósitos del trabajo con los proyectos de los profesores en formación de la carrera de Lenguas Extranjeras están aquellos vinculados a la actividad investigativa: preparar a los estudiantes para aprender contenidos de otras materias a través del idioma extranjero, exponer a los estudiantes a nuevos contenidos a través del uso de diferentes fuentes de información y promover en los estudiantes la reflexión y la autoevaluación de su propio aprendizaje y el de sus compañeros a partir del rol activo que deben asumir en la definición y control de los criterios evaluativos que guiarán todo el proceso.

Las autoras de este trabajo consideran que en el trabajo con los proyectos, el profesor debe dar cumplimiento a otros propósitos vinculados a las habilidades investigativas y que son necesarios en un profesional de la educación. Entre ellos está desarrollar la habilidad de hacer fichas bibliográficas y de contenido. El estudiante debe fichar la bibliografía utilizada, consignarla correctamente y acreditar la bibliografía de la que tomó información. Es importante también que el estudiante procese la información teórica obtenida a partir de la bibliografía estudiada, que seleccione los métodos empíricos necesarios para obtener la información precisa y que procese los resultados obtenidos, así como que realice el diseño correcto de los instrumentos aplicados y que consigne los datos cuantitativos obtenidos tanto en tablas como en gráficos.

El trabajo con proyectos en la disciplina Práctica Integral de la Lengua Inglesa y de la Lengua Francesa en los diferentes años permite el desarrollo de manera natural de las habilidades lingüísticas tales como la habilidad de comprensión lectora, de expresión oral, de comprensión y de escritura. Dentro de ésta, el uso del lenguaje científico y las habilidades académicas de resumir y parafrasear tienen un rol protagónico.

La habilidad de comprensión lectora aunque es una actividad receptiva y esencialmente silente, se caracteriza por ser un proceso activo y de interacción comunicativa entre escritor y lector, donde el lector tomará la información que necesita haciendo uso del resumen y la paráfrasis. Muchas de las habilidades lectoras son cardinales en el proceso investigativo, y entre las que se subrayan las siguientes:

. Identificar las ideas centrales (apartados textuales)

Reconocer y recordar detalles específicos

. Reconocer la relación que existe entre las ideas centrales y sus ampliaciones

. Leer entre líneas (inferencia de ideas)

- Llegar a conclusiones

. Reconocer la actitud y el propósito del escritor (para adscribirse a uno o a otro autor)

. Extraer los puntos que sobresalen (información relevante) para resumir (un texto, una idea, etc.) y tomar nota de ello.

El desarrollo de la habilidad de expresión oral es muy importante en la investigación por cuanto el investigador expone los resultados de su trabajo, realizado éste ya sea en inglés o en francés según corresponda. Para estas actividades se necesita de precisión y fluidez en el idioma así como el uso del lenguaje científico donde las formas de elocución más frecuentes son la exposición y argumentación, usadas de forma combinada. En cuanto a la habilidad de expresión escrita, es necesaria la referencia de diferentes tipos de modelos que se trabajan en la clase de Práctica Integral de la Lengua Inglesa y Francesa tales como: Ilenado de modelos, 
notas, correos electrónicos cartas, redacción de diferentes formas de elocución entre las que se destacan la descripción, narración, argumentación, redacción de ensayos, etc. Cuando el alumno escribe, se establecen las diferencias entre el lenguaje oral y el escrito, y se exige a los estudiantes cierto nivel de corrección sintáctica, con un lenguaje claro y conciso haciendo uso de las oraciones de coordinación, yuxtaposición fundamentalmente. Todas estas exigencias acercan al estudiante al uso del lenguaje científico.

Entre las habilidades académicas se hace referencia al parafraseo y al resumen. La paráfrasis consiste en expresar los contenidos de un texto con las propias palabras con organización, continuidad y coherencia, las cuales deben representar el pensamiento y los principales contenidos propuestos por el autor de un texto. Esta técnica sirve para verificar lo que han comprendido y ayuda a los estudiantes en su habilidad para resumir y hacer inferencias porque deben traducir el pensamiento del autor de manera personal utilizando vocabulario y sintaxis propios. Resumir es expresar lógicamente con las propias palabras las ideas principales, relevantes que un texto presenta. http://www.educ.ar).

Durante la realización del trabajo científico estudiantil, los alumnos deberán consultar y leer textos informativos y narrativos necesitarán contar con los medios necesarios para desarrollar la habilidad de registrar y reorganizar los elementos del texto, mostrando dominio del contenido para luego consignarlo por escrito.

Las habilidades investigativas son bases del aprender a investigar, y el desarrollo de estas habilidades presupone en este proceso el aprendizaje de mecanismos que optimizan la actividad de estudio, la actividad investigativa, la auto regulación en el proceso, el fomento de valores, el desarrollo de actitudes y disposiciones para el aprendizaje que potencian la capacidad para el auto desarrollo. La práctica pedagógica ha demostrado que se hace necesario el desarrollo en los profesionales en formación de cualidades que los distingan en su formación investigativa. Éstos deben superar la concepción sobre el pensar y el pensamiento como solo aprender a rememorar o aprender a repetir o aprender a demostrar la demostración de otros. Deben superar la concepción del hacer como solo hacer un trabajo o hacer una tarea o hacer una copia de los saberes ya dados. Deben superar la concepción sobre el ser como solo a ser imitación dependiente de otro o ser valoración por subordinación de sus propios valores o a ser interdependencia o ser valoración por autoritarismo sin relación autónoma. La imaginación y la creatividad no solo son parte fundamental de la estructura de conciencia interna del sujeto investigador sino la interrelación que conlleva a interpretar la realidad valiéndose de innovaciones que permitan crear una atmósfera productiva y novedosa.

\section{Conclusiones}

Los proyectos realizados por el profesor en formación de lenguas extranjeras son actividades que promueven el aprendizaje cooperativo y permiten el desarrollo de habilidades comunicativas y de modos de actuación para la investigación como parte de la formación de este profesional.

Habilidades comunicativas como la comprensión lectora, expresión oral, expresión escrita, de escucha y otras académicas como el resumen y el parafraseo contribuyen a sentar bases necesarias para el desarrollo de habilidades investigativas que juegan un rol esencial en la formación de modos de actuación para la actividad investigativa y para la cultura científicoinvestigativa de este profesional. 


\section{Bibliografía}

Álvarez de Zayas, C. Hacia una escuela de excelencia Académica. La Habana. 1996.

Barrio, M. Las cuerdas locuras del cambio. Entrevista concedida por el Dr. Luis I. G. Gutiérrez. Ministro de Educación. En Juventud Rebelde, 31-3-2002.

Belyayev, B.V. Psychology of Teaching Foreign Languages. Pergamon Press. Moscú, 1963.

Brandani, L. ¿Cómo hacer un resumen? /Lucía Brandani / Juan Rearte. [En Línea]. Disponible en http://www.educ.ar Consultado el 18 de febrero de 2012.

Brinton, D.M. y Master, P. New Ways in Content-Based Instruction. TESOL. Alexandria. Estados Unidos de América, 1997.

Brumfit Ch. Communicative Methodology in Language Teaching: The role of fluency and accuracy. Cambridge University Press, Londres, 1984.

Camacho A. The Content of EFL at the Secondary School Level in Cuba. (Ponencia inédita) Santa Clara, 2001.

Castellano, S. D. y otros. Aprender y enseñar en la escuela: una concepción desarrolladora. Editorial pueblo y educación. Ciudad de la Habana. 2002

Colectivo de Autores. Tendencias Pedagógicas Contemporáneas. Ediciones ENPES. La Habana, 1991.

Colectivo de Autores. Metodología de la investigación educacional. Desafíos y Polémicas actuales. Editorial Pueblo y Educación. La Habana (coma) 2005.

Fried-Booth, D. y Maley, A. Project Work. Cambridge University Press, 1986.

Fried-Booth, D. y Maley, A. Project Work. Oxford University Press, 2002.

Horroutininer Silva, P. La Universidad Cubana: el modelo de formación. 2003

Martí, José. Obras Completas. La Habana, Editorial de Ciencias Sociales, 1975.

Martínez Llantada, M. Calidad Educacional. Actividad pedagógica y creatividad. Editorial Academia. La Habana, 1998.

\section{Notas}

${ }^{1}$ Martí, José. Obras Completas. La Habana, Editorial de Ciencias Sociales, 1975. -- t.XII, p.375

Fecha de recibido: 21/01/2013

Fecha de aceptación: 10/06/2013

\footnotetext{
* M.Sc. en Estudios de lengua Inglesa, Universidad Central Martha Abreu, Cuba. Profesora Superior en Inglés, Universidad Central Martha Abreu, Villa Clara, Cuba. Miembro del Consejo Científico de la Facultad de Humanidades.

** Master en Teoría y Práctica de la Enseñanza del Inglés Contemporáneo. Licenciada en Educación. Especialidad Lengua Inglesa, Universidad Félix Varela Morales. Profesora, Universidad de Ciencias Pedagógicas de Villa Clara.

*** Master en Teoría y práctica de la enseñanza de la Lengua Inglesa, Universidad Félix Varela Morales. Licenciada en Educación. Especialidad Lengua Inglesa, Universidad Félix Varela Morales. Jefa del departamento de profesores de Lenguas Extranjeras de la Universidad Pedagógica Félix Varela Morales de Villa Clara.
} 